\title{
Testing of Thin Permanent Magnets at Higher Frequencies
}

\author{
Dominik Perchtold ${ }^{1}$, Manfred Kaltenbacher ${ }^{1}$, Hendrik Husstedt ${ }^{2}$ \\ 1 Vienna University of Technology, Getreidemarkt 9, 1060 Wien, Austria, \\ dominik.perchtold@tuwien.ac.at \\ 2 Deutsches Hörgeräte Institut GmbH, Bessemer Straße 3, 23562 Lübeck, Germany
}

\begin{abstract}
In this work, we present an application-oriented method to characterize permanent magnets at higher frequencies. The main idea is to identify the permeability and the remanence of a sample without measuring the complete hysteresis curve. Therefore, the remanence is determined by using a magnetic equivalent circuit, whereas the permeability can be measured directly. This requires more quantities to be measured but results in less field intensity needed. Several aspects of the design of the measurement setup and the evaluation of the acquired data are presented.
\end{abstract}

Key words: permanent magnets, characterization, finite element analysis, electromagnetics.

\section{Introduction}

In this paper, an application-oriented approach to characterize permanent magnet (PM) samples is presented. Although there are standard methods, as defined in the IEC 604045 , and commercially available equipment with established methods, see e.g. [1][2][3], we developed a measurement setup to meet our requirements. The reasons for this are presented in the following.

In most industrial applications, only the linear range of the operating characteristics of a PM is of interest. If a PM is operated outside these characteristics, it can be depolarized or the application is affected in a way that it is nonfunctional. This means that there is no need to measure the complete hysteresis curve of a sample, as only the linear permeability and the remanence are interesting. Additionally, the complete characterization requires high field intensities.

In the IEC 60404-5, the minimum dimension of the sample is specified as $5 \mathrm{~mm}$. The samples, which we are interested in, have a length of $1.5 \mathrm{~mm}$ in the direction of the polarization. Additionally, the samples should be characterized at frequencies up to $20 \mathrm{kHz}$. Since massive cores are needed to provide a homogeneous magnetic field, where the sample can be placed, the inductance of such a setup is typically high. This results in high requirements for the power supply to drive the excitation coils at higher frequencies.

Another important reason for the development of this solution is that the same samples can be used in the application as in the measurement without the need for samples of different dimensions. Moreover, different magnetic load scenarios in the linear range can also be applied by the measurement setup itself. This enables the characterization during stress tests with the measurement setup or the characterization before and after stress tests in the application.

In order to avoid massive, costly and highly specialized hardware, a custom solution of a closed-loop measurement principle has been developed.

\section{Measurement Setup}

The sample under test is placed in a closed magnetic loop between two U-shaped ferrite cores, as depicted in Fig. 1. This core material is chosen because of its low electric conductivity of about $0.2 \mathrm{~A} /(\mathrm{Vm})$ according to the data sheet, which makes it suited for higher frequencies.

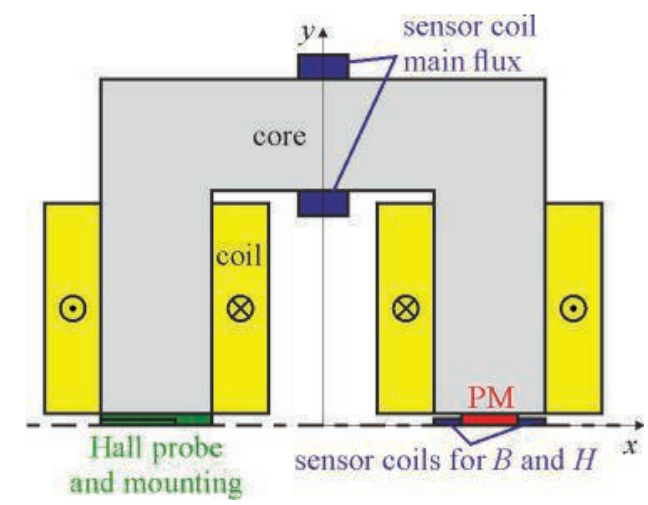

Fig. 1. Draft of the measurement setup.

Since the sample is characterized by measuring only a part of its linear characteristics, more 
quantities have to be measured in comparison to the standard IEC 60404-5. The magnetic flux is measured by sensing coils at three different positions. First, the main flux through the core, second, the flux through the PM, and third, the flux alongside the PM. In the second air gap of the setup, a Hall probe is placed. The mounting containing the Hall probe is also used for aligning the cores.

One of the sensing coils encloses the PM, which allows for calculating the mean flux density in the PM. The second sensing coil encloses only air near the PM, which enables measuring the mean field intensity in the sample due to the continuity of the tangential component of the magnetic field intensity.

\section{Design}

As the ferrite core has a low conductivity but also a low saturation flux density compared to the remanence of the samples, the design becomes important. With the help of finite element (FE) simulations, several investigations have been made concerning the width of the core, the combination of different materials, and the measurement of the magnetic field intensity. The width of the core mainly affects the inductance of the setup. Although a small inductance is desirable due to the excitation at higher frequencies, the core must be wide enough to guarantee a homogeneous field distribution. Additionally, it must provide enough space to place sensing coils, as depicted in Fig. 2. The samples under test have dimensions of $8.3 \mathrm{~mm} \times$ $25 \mathrm{~mm} \times 1.5 \mathrm{~mm}$.

The field intensity is the most important quantity to be considered because it does not only determine the precisely measurable range but it also affects the combination of different materials of the core and the sample. In order to demonstrate the measurable range, the field intensity across the air gap is shown in Fig. 3, which is obtained from 3D FE simulations. To sum up, the field intensity has to be the same inside and alongside the PM in order to determine it correctly.

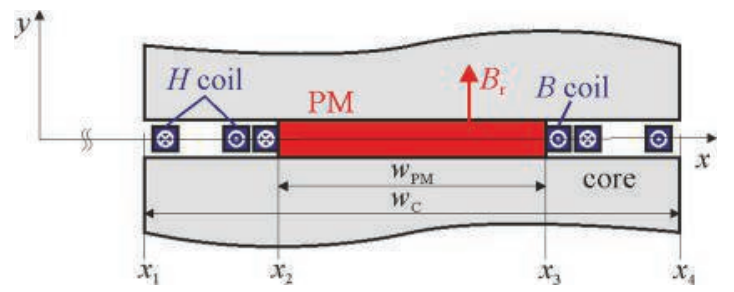

Fig. 2. Details of the air gap with the sample under test.

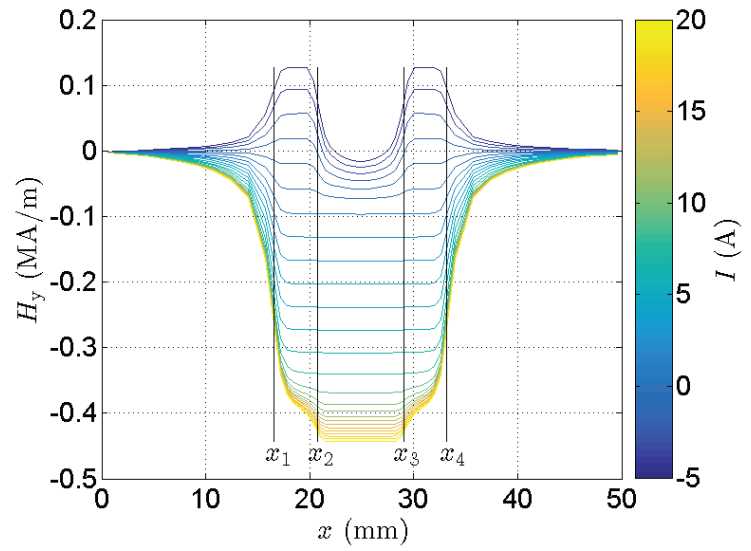

Fig. 3. Magnetic field intensity across the air gap with the sample under test.

The field intensity inside and alongside the sample is shown in Fig. 4 against different excitation currents. An overview of the difference between the field intensities with different materials is shown in Fig. 5. It depicts the relative difference between the field intensity inside and alongside the sample under test against the field intensity inside the PM. The first case is the combination chosen for constructing the measurement setup. It combines the ferrite material 3C90 [5] with the samples which are to be characterized, namely plastic-bonded Neodymium magnets of the type $N d F e B 55 / 100$ pw [4]. The second case shows the effects of using a sintered PM of the type Sm2Co17 180/160w [6] with a much higher remanence. The third case involves a powder-core of the type KoolMu 60 [7] with a low permeability but high saturation. Last, the results of a laminated sheet-core of the type VACOFLUX50 [8] are shown, which has a comparably high permeability and saturation. It is shown that the permeability and the saturation of the core must be high enough depending on the samples to be investigated.

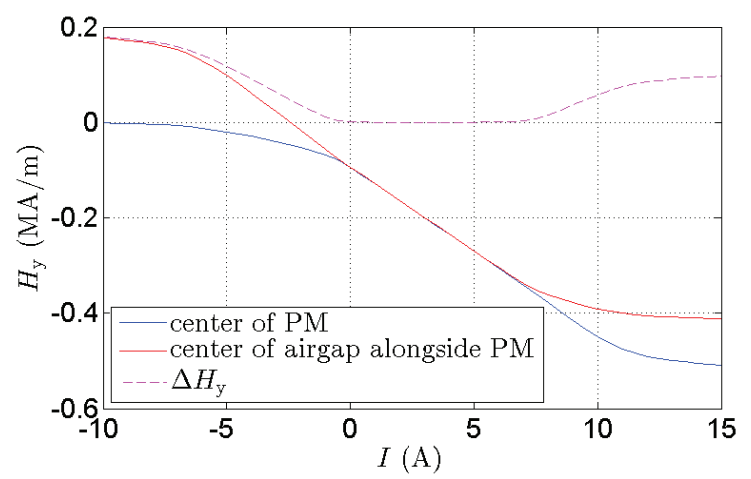

Fig. 4. Field intensity inside and alongside the PM against excitation current with the core material 3 C90 and an NdFeB 55/100 pw sample. 


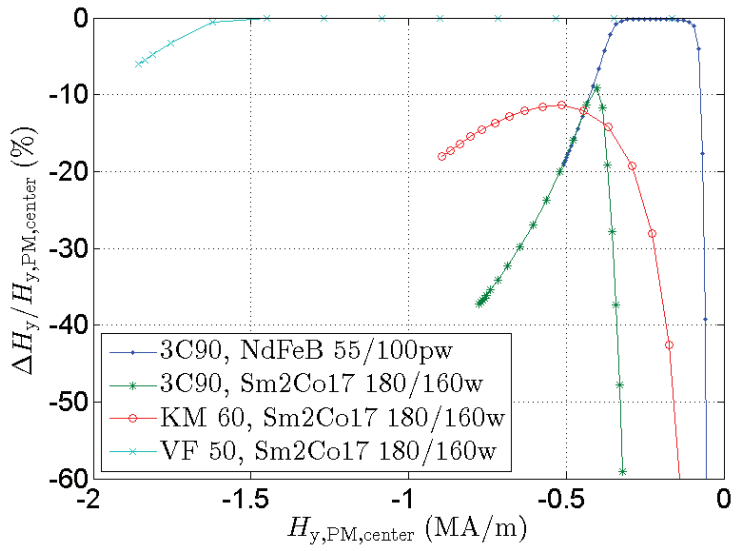

Fig. 5. Relative difference between the field intensity inside and alongside the PM for different materials combinations of the core and the PM.

\section{Evaluation of Measurements}

Since the goal is to characterize the permeability and the remanence of the PM in the linear range without measuring the complete hysteresis curve, the remanence has to be determined by calculating the operating point. Therefore, the Hall probe is required because it provides the DC component of the flux, whereas the sensing coils only measure $\mathrm{AC}$ components. For this purpose, the magnetic equivalent circuit as depicted in Fig. 6 is considered, where $R_{\text {Hall }}$ models the air gap with the Hall probe, $R_{\mathrm{C}}$ the resistance of the core, $R_{\mathrm{S} 1}$ and $R_{\mathrm{S} 2}$ the stray resistances, and the $\mathrm{PM}$ is modeled by the flux source with the inner resistance $R_{\mathrm{PM}}$. The magnetic voltage source $\mathrm{Ni}$ models the excitation coils.

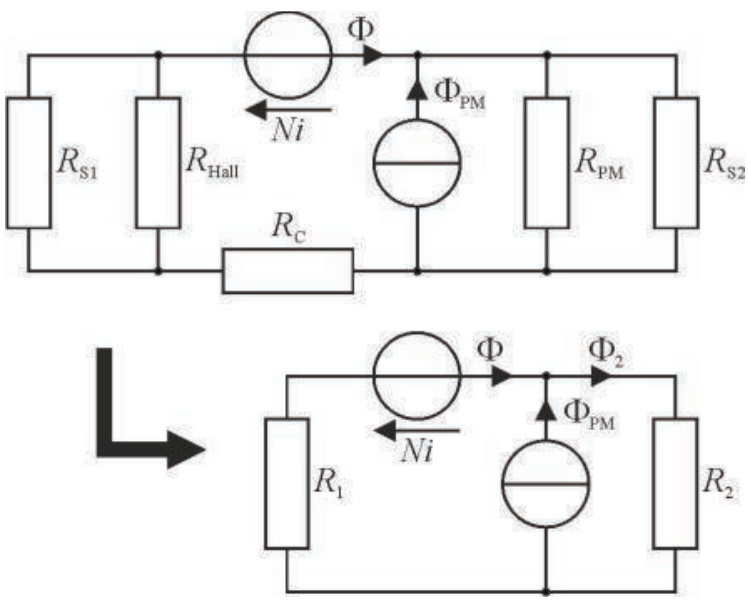

Fig. 6. Magnetic equivalent circuit and magnetic resistors considered for the identification.

The idea is to identify the combined magnetic resistors modeling the air gaps and stray fluxes from the AC signals. Since we are interested in the linear characteristics and the measurable range of $H$ is known, the whole circuit is assumed linear in the corresponding range. This enables the calculation of the operating point with the help of the Hall probe after the identification of the magnetic resistors.

The total magnetic resistance and the resistance of the air gap with the sample under test can be calculated from the AC amplitudes of the current, of the main flux and of the field intensity near the sample. This can be expressed in terms of the equivalent circuit as

$R_{\text {tot }}=R_{1}+R_{2}=N \hat{I} / \widehat{\Phi}, R_{2}=\widehat{H}_{2} l_{2} / \widehat{\Phi}$,

since the PM does not contribute to the $A C$ quantities. Next, the DC component of the main flux has to be calculated. Therefore, a relation between the main flux and the flux density measured by the Hall probe is required. From the $A C$ quantities, the linear factor can be obtained as

$\alpha=\widehat{\Phi} / \widehat{B}_{\text {Hall }}$.

Taking the DC components into account, the relation becomes non-linear and can be written as

$\Phi=\alpha B_{\text {Hall }}+\beta\left(B_{\mathrm{r}}\right)$.

This offset has to be taken into account in any case, regardless whether the core is saturated in the operating point or not. It has to be determined in order to solve the equations obtained from the equivalent circuit. In the example presented, the offset is calculated from 3D FE simulations for a small range around the nominal remanence, which is depicted in Fig. 7.

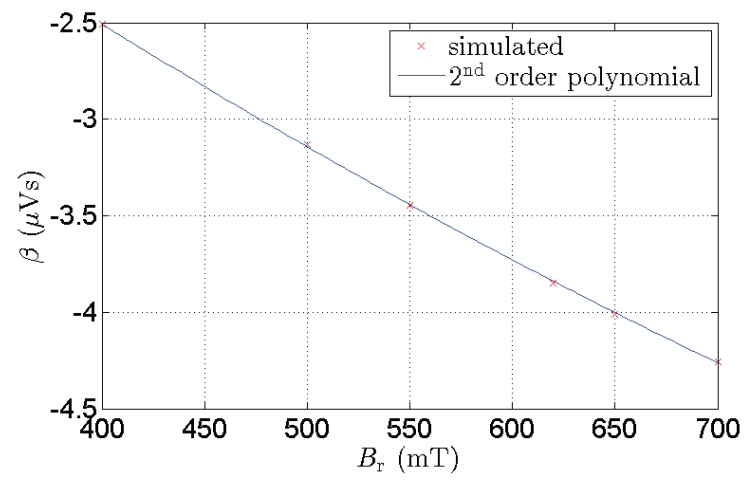

Fig. 7. Calibration offset obtained from $3 D$ FE simulations.

Using relation (3), the flux provided by the PM can be calculated as

$\Phi_{\mathrm{PM}}=-\left.\Phi\right|_{i=0} R_{\mathrm{tot}} / R_{2}$,

which also means that the excitation current has to cross zero.

\section{Results}

An example characterization is shown in Fig. 8, which demonstrates the applicability and shows where the measurement of the field intensity is limited due to the saturation of the core. The 
visible saturation effects are not caused by the sample.

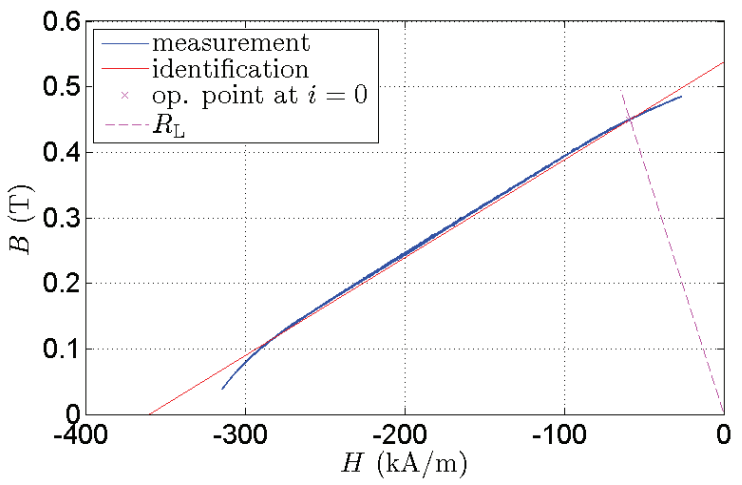

Fig. 8. Example characterization of a Neodymium sample demonstrating the saturation of the core.

Concerning the non-linearity and the phase shift of the AC signals, we want to describe the identification of the magnetic resistors is in more detail. Since we are only interested in the amplitudes, the phase differences are corrected. In practice, this is done by fitting a sine function with an offset to the measured data in the linear range, i.e. neglecting all points in the non-linear region. The phase information can be used to shift all signals to the phase of one of the quantities, e.g. the main flux. This is important for the direct evaluation of the permeability, as well as for relation (3). In order to demonstrate the effects of the phase correction, the identification of the resistors according to (1) is shown in Figures 9 and 10 for two different frequencies. The identification works correctly, whereas the measurement signals show different phase shifts, which lead to ellipse-shaped characteristics of the magnetic flux against the magnetic voltage.

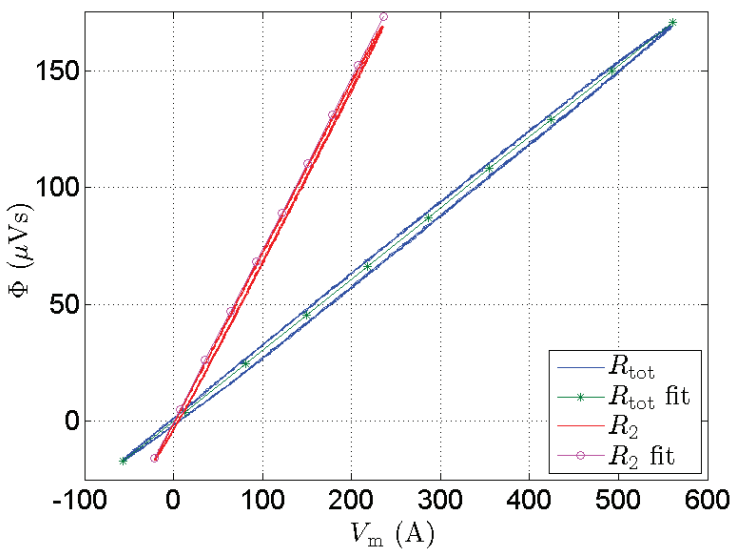

Fig. 9. Measurement data and identification of the magnetic resistors at $1 \mathrm{~Hz}$.

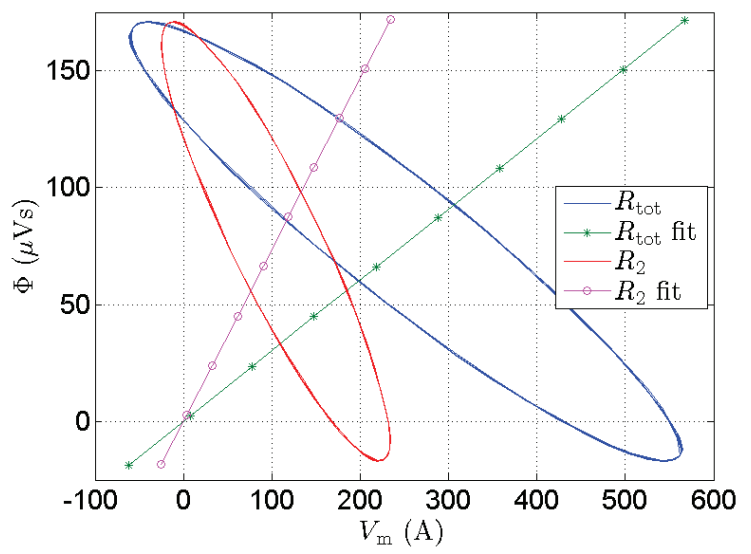

Fig. 10. Measurment data and identification of the magnetic resistors at $2 \mathrm{kHz}$.

Another experiment shows the influence of the temperature. In Fig. 11, the measurement signals of the $\mathrm{B}$ and $\mathrm{H}$ coils are displayed. As the core is also affected by temperature, the linear range, which can be exploited, becomes smaller with rising temperature. The smaller permeability and saturation of the core lead to an error of the measured field intensity, similar to the results shown in the design section. However, the designed setup is functional up to the maximum operating temperature of the sample, which is $130^{\circ} \mathrm{C}$. It must be noted that the sample was not severely affected irreversibly by the relatively short time at about $200^{\circ} \mathrm{C}$ and its Curie temperature is at $310^{\circ} \mathrm{C}$. This leads to the conclusion that the measured curves are indeed limited by the core material.

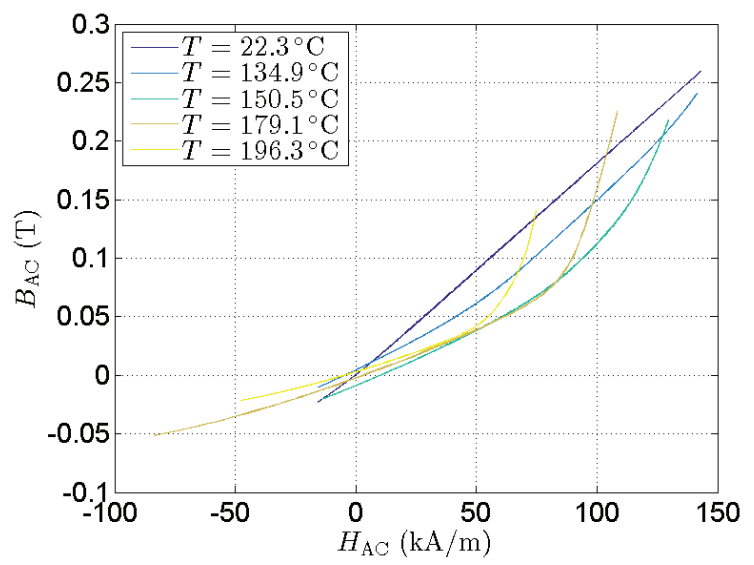

Fig. 11. Measured flux density and field intensity at higher temperatures.

\section{Conclusion}

A method is presented to identify the permeability and remanence of a permanent magnet (PM) while only measuring a part of its linear characteristic. Compared to measuring the full hysteresis curve, the field intensity applied to the PM is lower, which results in less power required for the supply of the excitation coils. 
The corresponding measurement setup is designed to minimize the effect of eddy currents in the core and to be functional up to the nominal operating temperature of the PM.

\section{References}

[1] F. Fiorillo, Measurement and Characterization of Magnetic Materials, Elsevier series in electromagnetism (2004)

[2] S. Liu and E. P. Hoffman, Application-Oriented Characterization of $\mathrm{Sm}_{2}(\mathrm{Co}, \mathrm{Fe}, \mathrm{Cu}, \mathrm{Zr})_{17}$ Permanent Magnets, IEEE Transactions on Magnetics, Vol. 32, No. 5 (1996)

[3] R. Cornelius et al., Pulsed Field Magnetometer for Industrial Use, IEEE Transactions on Magnetics, Vol. 38, No. 5 (2002)

[4] Data sheet of NdFeB 55/100 pw, MSSchramberg GmbH \& Co. KG, 78713 Schramberg-Sulgen, Germany (2010)

[5] Data sheet of 3C90, Ferroxcube Headquarters, New Taipei City 23145, Taiwan (2010)

[6] Data sheet of Sm2Co17 180/160 w, MSSchramberg GmbH \& Co. KG, 78713 Schramberg-Sulgen, Germany (2010)

[7] Data sheet of KoolMu 60, Magnetics Headquarters, Pittsburgh, PA 15238, USA (2014)

[8] Data sheet of VACOFLUX50, VACUUMSCHMELZE GmbH \& Co. KG, 63450 Hanau, Germany (2001) 